\title{
Annular Iris Recognition Using SURF
}

\author{
Hunny Mehrotra ${ }^{1}$, Banshidhar Majhi ${ }^{1}$, and Phalguni Gupta ${ }^{2}$ \\ 1 National Institute of Technology Rourkela, Rourkela 769008 \\ 2 Indian Institute of Technology Kanpur, Kanpur 208016 \\ \{hunny, bmajhi\}@nitrkl.ac.in, pg@iitk.ac.in
}

\begin{abstract}
This paper proposes an iris recognition system which can handle efficiently the problem of rotation, scaling, change in gaze of individual and partial occlusions that are inherent to non-restrictive iris imaging system. In addition to this, traditional iris normalisation approach deforms texture features linearly due to change in camera to eye distance or non-uniform illumination. To overcome the effect of aliasing features are extracted directly from annular region of iris using Speeded Up Robust Features (SURF). These features are invariant to transformations and occlusion. The system is tested on BATH, CASIA and IITK databases and is showing an accuracy of more than $97 \%$. From the results it is inferred that local features from annular iris gives much better accuracy for poor quality images in comparison to normalised iris.
\end{abstract}

Keywords: Annular Iris Region, SURF, Local Features, Occlusion, Transformation.

\section{Introduction}

Iris is gaining added attention since last few decades due to accuracy, reliability and speed. Iris image acquisition is a highly restrictive process that requires cooperative and well trained audience. Acquired image is localised using pupil and iris boundary. Further, segmented iris region is normalised to form a rectangular image for matching. However there are several issues to be taken into consideration prior to feature extraction and matching. Some of these issues are worth to mention. During image acquisition, there may be some tilt in head or change in gaze of an individual. Thus the features are transformed circularly in Cartesian plane. Again iris image may be occluded by lower and upper eyelids that makes images inappropriate for matching. Another issue is that texture pattern in iris undergoes linear deformation due to expansion and contraction of pupil under non-uniform illumination. Further mapping from Cartesian to polar plane creates the effect of aliasing that loses significant texture details that are relevant from recognition point of view.

There exists several global feature extraction techniques in iris. In [1 Gaussian filter at multiple scales is used to extract features. Iris coding method based on differences of Discrete Cosine Transform (DCT) coefficients of overlapped angular patches from normalised iris images is presented in [2]. In [3] wavelet transform is applied on circular bands of iris and zero crossing representation is used

S. Chaudhury et al. (Eds.): PReMI 2009, LNCS 5909, pp. 464-469, 2009.

(C) Springer-Verlag Berlin Heidelberg 2009 
for coding. These approaches extract global features and works on normalised iris image. Global feature extraction techniques generally fail under change in illumination, rotation and occlusion. To make a robust iris recognition system, local features can be extracted around the keypoints detected from annular iris image. These features are based on object representation using interest points and possess invariance to rotation, scaling, illumination and performs with certain degree of occlusions as well. There has been considerable amount of work done for object localisation and biometrics recognition using keypoint descriptors 4 [5. These local descriptors have been applied on iris recognition as well. A novel corner point descriptor is developed for iris that uses Harris corner detector to extract keypoints and entropy information of window around the corner as descriptor [6. However, Harris corners are not scale invariant and fails to achieve property of repeatability when iris deforms due to illumination. Further, region based Scale Invariant Feature Transform (SIFT) has been applied on annular portion of iris. The system is designed for non-cooperative iris database [7.

This paper proposes an iris recognition system which makes an attempt to handle the above mentioned issues. To avoid loss of information due to mapping, segmented iris image (annular region) is considered directly for feature extraction without normalisation as given in Section 2. Further local features are extracted from segmented annular region to overcome the effect of occlusion, rotation and illumination. In this paper, Speeded Up Robust Features (SURF) 8] are used for feature extraction as given in Section 3. SURF uses same matching approach as SIFT but with few variations. Firstly, SURF uses sign of Laplacian to have sharp distinction between background and foreground features. Secondly, SURF uses only 64 dimensions compared to SIFT using 128 dimensional vector. This reduces feature computation time and allows quick matching with increased robustness simultaneously. After feature extraction, two iris images are paired using matching strategy given in Section 4. The results of the proposed system has been analysed in Section 5. Conclusion is given in the last section.

\section{Iris Preprocessing}

The acquired iris image contains some irrelevant information that has to be removed prior to feature extraction. Preprocessing involves detection of inner and outer iris boundary using Circular Hough Transform (CHT) [9] as shown in Fig. 1(a). Light spots on pupil region adds noise and impediments the localization process. These spots are detected and filled using morphological region filling algorithm [10]. Due to variation in illumination and change in camera to eye distance the annular region lying between pupil and iris boundary is highly variable. In order to overcome these variations traditional iris recognition approaches transforms annular region into polar coordinate. However, Hugo et. al in [11] have studied the problem of aliasing that occurs during polar transformation. The authors have studied the relationship between size of captured iris image and recognition accuracy. It has been inferred that due to change in 
area the recognition accuracy reduces considerably. In the proposed paper the problem of aliasing is removed by considering the annular region of iris without normalisation. After localisation of inner and outer iris boundary iris ring is segmented from the rest of the image as shown in Fig. 1(b).

\section{$3 \quad$ Feature Extraction Using SURF}

Global feature extraction techniques fail to work directly on annular iris images. The performance of feature extractor lies in its ability find the same image despite of change in scale, rotation and illumination. Local features are extracted by finding the keypoints in an image and forming descriptor vector around each detected keypoint. These features renders small changes in the descriptor with significant change in relative position of keypoints. Hence descriptors are paired even with affine distortions and occlusions of keypoints. SURF is a descriptor that extracts distinctive and stable features with relatively less computational requirements [8]. Steps involved to extract features using SURF are

\subsection{Keypoint Detector}

For fast detection of keypoints Gaussian second order derivative is used with box filters, in contrast to SIFT that approximates Laplacian of Gaussian (LOG) with Difference of Gaussian (DOG). For each pixel $p$ in an image, Hessian matrix for $p$ at scale $\sigma$ is obtained. The determinant of Hessian matrix is used for selecting location and scale. The local maxima found of the approximated Hessian matrix determinant are interpolated in scale and image space. The detected keypoints on iris is shown in Fig. 1(c).

\subsection{SURF Descriptor}

A circular window is constructed around every detected keypoint and orientation is estimated using Haar Wavelet responses to have invariance to rotation. Further, SURF descriptors are obtained by taking a rectangular window around every detected keypoint in the direction of orientation. The windows are split into $4 \times 4$ sub regions. For each sub region Haar wavelet responses are extracted for equally spaced sample points 12 . Finally the wavelet response in horizontal $\left(d_{x}\right)$ and vertical $\left(d_{y}\right)$ direction are summed up for each sub region. The absolute values of wavelet responses $\left(\left|d_{x}\right|\right.$ and $\left.\left|d_{y}\right|\right)$ are summed up to find the polarity of image intensity changes. Hence feature vector for each sub region is given by

$$
\mathrm{fv}=\left(\sum d_{x}, \sum d_{y}, \sum\left|d_{x}\right|, \sum\left|d_{y}\right|\right)
$$

Thus summing up the descriptor vectors from all $4 \times 4$ sub-regions results in feature descriptor of length 64 . The descriptor vector of length 64 for each interest point forms the feature vector. 


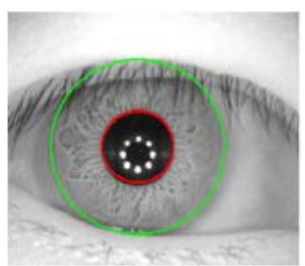

(a)

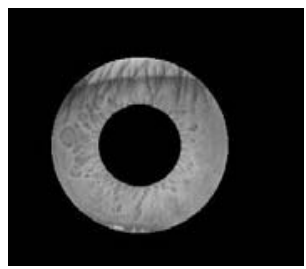

(b)

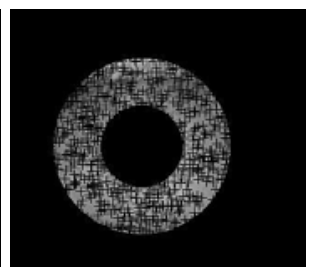

(c)

Fig. 1. Steps involved in proposed iris recognition system (a) Iris localisation using Hough transform (b) Annular segmentation of iris (c) Detection of keypoints on annular region

\section{Iris Pairing}

After detection of keypoints in gallery image $(A)$ and probe image $(B)$, matching is done using interest point pairing approach. The best candidate match for each keypoint in $A$ is found by identifying the closest pair from the set of keypoints in $B$. The nearest neighbor is defined as the keypoint with minimum Euclidean distance for the invariant descriptor vector. Let $L=\left\{l_{1}, l_{2}, l_{3} \ldots . l_{m}\right\}$ and $E=\left\{e_{1}, e_{2}, e_{3} \ldots . e_{n}\right\}$ be vector arrays of keypoints of $A$ and $B$ respectively. The descriptor array $l_{i}$ of keypoint $i$ in $L$ and descriptor array $e_{j}$ of keypoint $j$ in $E$ are paired if the Euclidean distance $\left\|l_{i}-e_{j}\right\|$ between them is less than a specified threshold $\tau$. Threshold based pairing results in several number of matching points. To avoid multiple matches, the keypoints with minimum descriptor distance and less than threshold are paired. This results in a single matching pair, and is called as nearest neighbourhood matching method. The paired points $\left(l_{i}\right.$, $e_{j}$ ) are removed from $L$ and $E$ respectively. The matching process is continued until there are no more keypoints in $L$. The number of paired points between sample images $A$ and $B$ is shown in Fig. 2

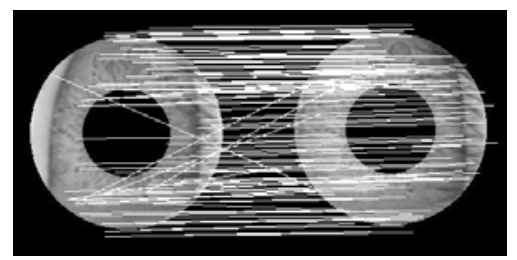

Fig. 2. Pairing between gallary and probe iris images

\section{Experimental Results}

The proposed system has used BATH [13, CASIA [14] and Indian Institute of Technology Kanpur (IITK) [15] databases to determine its performance. Database available from BATH University comprises of 20 images from both 
Table 1. Results generated using SURF for normalized and annular iris image (values in $\%$ )

\begin{tabular}{|l|c|c|c|c|c|c|c|c|c|}
\hline Databases $\rightarrow$ & \multicolumn{3}{|c|}{ BATH } & \multicolumn{3}{c|}{ CASIAV3 } & \multicolumn{3}{c|}{ IITK } \\
\hline Testcases $\downarrow$ & FAR & FRR & Accuracy & FAR & FRR & Accuracy & FAR & FRR & Accuracy \\
\hline Normalized Iris & 10.35 & 21.11 & 84.26 & 3.31 & 5.13 & 95.77 & 0.86 & 5.52 & 96.80 \\
\hline Annular Iris & 2.37 & 1.97 & 97.84 & 1.44 & 4.07 & 97.23 & 4.65 & 1.41 & 97.15 \\
\hline
\end{tabular}

the eyes for 50 subjects $(50 \times 20)$. CASIA-IrisV3 database comprises of 249 subjects with total of 2655 images from both the eyes. The database collected at IITK consists of over 1800 iris images taken from 600 subjects $(600 \times 3)$ only from left eye. To measure the performance of the proposed system, genuine and imposter scores are generated using three databases. Distribution of genuine and imposter scores for BATH is given in Fig. 3(a). Similar curves are obtained for CASIA and IITK databases. To measure the robustness of the system, ROC curves are obtained for all the three databases as shown in Fig. 3(b). Table 1 provides comparative performance of SURF using two different testcases i.e., (a) normalized and (b) annular iris images. From the results it is evident that the system performs well in both the testcases for IITK database where the acquired image is of sufficiently large size and covers most part of the eye. Thus the problem of aliasing does not arise and hence traditional normalisation technique gives equally good results. However for BATH database the images are distant from the camera with less iris details. Traditional normalisation in case of BATH creates aliasing effect and shows poor performance with very low accuracy of $84.26 \%$. However, using annular iris image the accuracy increases significantly to $97.84 \%$. Similarly, the accuracy of CASIA database increases by $2 \%$.

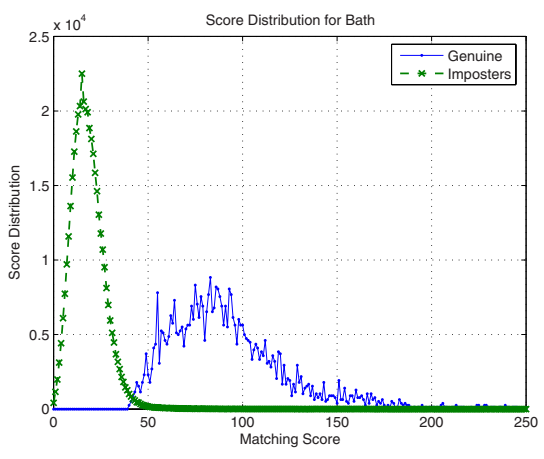

(a)

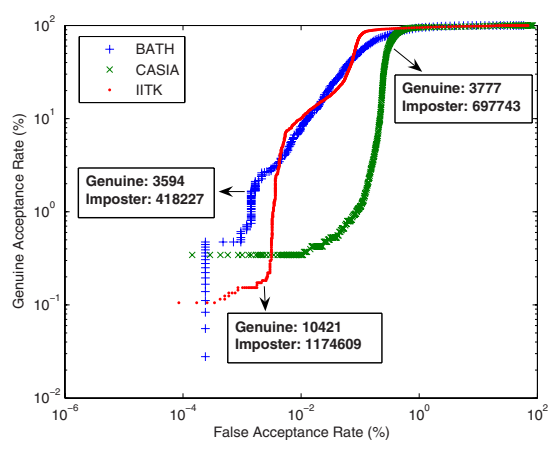

(b)

Fig. 3. Performance results of annular iris recognition: (a) Distribution of genuine and imposter scores for BATH database (b) ROC curves for BATH, CASIA and IITK databases 


\section{Conclusion}

This paper has proposed an iris recognition system that works under unconstrained imagining conditions. In this approach the problem of aliasing that occurs using traditional normalisation approach has been addressed by extracting features directly from annular iris image. Feature extraction using iris circle is a challenging task. Thus, SURF is used to extract local features having invariance to basic transformations. The system has been tested against standard available databases with and without normalisation. It has been found that the system performs better for all kinds of iris imagery with robustness to rotation and linear deformations that are inherent to iris.

\section{References}

1. Daugman, J.G.: High confidence visual recognition of persons by a test of statistical independence. IEEE Transactions on Pattern Analysis and Machine Intelligence 15(11), 1148-1161 (1993)

2. Monro, D.M., Rakshit, S., Zhang, D.: Dct-based iris recognition. IEEE Transactions on Pattern Analysis and Machine Intelligence 29(4), 586-595 (2007)

3. Boles, W.W., Boashash, B.: A human identification technique using images of the iris and wavelet transform. IEEE Transactions on Signal Processing 46(4), 11851188 (1998)

4. Lowe, D.G.: Distinctive image features from scale-invariant keypoints. International Journal of Computer Vision 60(2), 91-110 (2004)

5. Bicego, M., Lagorio, A., Grosso, E., Tistarelli, M.: On the use of sift features for face authentication. In: Conference on Computer Vision and Pattern Recognition Workshop, June 2006, pp. 35-35 (2006)

6. Mehrotra, H., Badrinath, G.S., Majhi, B., Gupta, P.: An efficient dual stage approach for iris feature extraction using interest point pairing. In: IEEE Workshop on Computational Intelligence in Biometrics: Theory, Algorithms, and Applications, April 2009, pp. 59-62 (2009)

7. Belcher, C., Du, Y.: Region-based sift approach to iris recognition. Optics and Lasers in Engineering 47(1), 139-147 (2009)

8. Bay, H., Ess, A., Tuytelaars, T., Gool, L.V.: Surf: Speeded up robust features. Computer Vision and Image Understanding (CVIU) 110(3), 346-359 (2008)

9. Kerbyson, D.J., Atherton, T.J.: Circle detection using hough transform filters. In: Fifth International Conference on Image Processing and its Applications, July 1995, pp. 370-374 (1995)

10. Gonzalez, R.C., Woods, R.E.: Digital Image Processing, 3rd edn. Prentice-Hall, Englewood Cliffs (2007)

11. Proenca, H., Alexandre, L.A.: Iris recognition: An analysis of the aliasing problem in the iris normalization stage. In: International Conference on Computational Intelligence and Security, vol. 2, pp. 1771-1774 (2006)

12. Bay, H., Fasel, B., Gool, L.V.: Interactive museum guide: Fast and robust recognition of museum objects (May 2006)

13. Bath University Database, http://www.bath.ac.uk/elec-eng/research/sipg/irisweb

14. Casia Database, http://www.cbsr.ia.ac.cn/english/Databases.asp

15. Database of Indian Institute of Technology Kanpur, http://www.cse.iitk.ac.in/users/biometrics 